\title{
Cognitive features of white matter lesions accompanied by different risk factors of cerebrovascular diseases
}

\author{
Yafei Shangguan ${ }^{\mathrm{A}, C-\mathrm{F}}$, Tao Xiong ${ }^{\mathrm{B}, \mathrm{C}, \mathrm{F}}$, Changwei Jiang ${ }^{\mathrm{C}, \mathrm{F}}$, Wei Chen ${ }^{\mathrm{C}, \mathrm{F}}$, \\ Yan Zhang ${ }^{B, F}$, Yongpin Zhao ${ }^{B, F}$, Guiyin Zhou ${ }^{C, F}$, Yulan Fan ${ }^{C}$, , Weimin Liu ${ }^{\mathrm{E}, F}$ \\ First People's Hospital of Guiyang, China \\ A - research concept and design; $\mathrm{B}$ - collection and/or assembly of data; $\mathrm{C}$ - data analysis and interpretation; \\ $D$ - writing the article; $E$ - critical revision of the article; $F$ - final approval of the article
}

Address for correspondence

Weimin Liu

E-mail:weiminliu7@163.com

\section{Funding sources}

This study was supported by Guiyang High-level Innovative Talents Project, project No. 27 ZWKJHTZDC [2013].

Conflict of interest

None declared

Received on June 4, 2018

Reviewed on January 8, 2019

Accepted on June 27, 2019

Published online on November 28, 2019

\section{Abstract}

Background. The relationship between different risk factors and the cognitive impairment of white matter lesions (WML) remains poorly understood.

Objectives. To investigate the features of cognitive impairment of patients diagnosed with WML accompanied by different risk factors of cerebrovascular diseases.

Material and methods. A total of 157 cases of WML patients were divided into no risk factor group $(n=26)$, hypertension group $(n=35)$, diabetes mellitus group ( $n=27)$, dyslipidemia group $(n=30)$, and mixed factors group $(n=39)$.

Results. The severity of WML (Fazekas score) in the hypertension and mixed factors groups was higher than in the non-risk factors group. The Montreal Cognitive Assessment (MoCA) scores in the hypertension and mixed factors groups were lower than in the non-risk factors group. The scores of MoCA, immediate memory and delayed recall in the hypertension and mixed factors groups with Fazekas score $\geq 3$ were lower than in the peer group with Fazekas score $<3$. The scores of MoCA and immediate memory in the hypertension and mixed factors groups with Fazekas score $\geq 3$ were lower than in the non-risk factors group with Fazekas score $\geq 3$.

Conclusions. Hypertension aggravates the severity of WML and cognitive impairment. The severity of WML is positively correlated with the severity of cognitive impairment accompanied by these risk factors.

Key words: cerebrovascular disease, cognitive impairment, atherosclerosis risk factors, white matter lesions

Cite as

Shangguan Y, Xiong T, Jiang C, et al. Cognitive features of white matter lesions accompanied by different risk factors of cerebrovascular diseases. Adv Clin Exp Med. 2019;28(12):1705-1710. doi:10.17219/acem/110324

D0I

10.17219/acem/110324

Copyright

Copyright by Author(s)

This is an article distributed under the terms of the

Creative Commons Attribution 3.0 Unported (CC BY 3.0)

(https://creativecommons.org/licenses/by/3.0/) 


\section{Introduction}

White matter lesions (WML), also known as leukoaraiosis (LA), are common imaging results and are correlated with the incidence of stroke, cognitive impairment, gait disorder, falls, depression, and death. ${ }^{1,2}$ They are associated with multiple pathologies, which include apoptosis, edema, widening of perivascular space, demyelination, axonal damage, gliocyte proliferation, and infarction. They are also accompanied by changes of the small blood vessels, such as fibrohyalinosis and venous collagenosis. The primary cause of WML is thought to be chronic ischemia. ${ }^{2}$ Hypertension, diabetes mellitus and hyperlipidemia, as well as WML itself, are risk factors for vascular cognitive impairment (VCI). ${ }^{3,4}$ Different risk factors can cause the formation of cognitive impairment with different mechanisms ${ }^{5-7}$ and may also exert different effects on cognitive function. However, the relationship between different risk factors and cognitive impairment of WML remains poorly understood. Therefore, this research focuses on WML patients presenting different risk factors of cerebrovascular diseases. Cranial imaging scale of cognitive function was used to analyze the features of cognitive functional impairment and determine the factors relevant for identifying and preventing cognitive impairment caused by WML during the early stage of cognitive impairment.

\section{Material and methods}

\section{Patients}

The general clinical characteristics of the patients and the related data of each group are presented in Table 1 . There were no statistical differences in terms of sex ratio, age, years of education, activities of daily living (ADL) scale, or Hamilton Depression (HAMD) scale among the different risk factor groups.

A total of 157 patients were enrolled, including 57 males and 100 females, aged $70.4 \pm 9.1$ years on average (range: $41-87$ years), with a mean education level of $9.8 \pm 4.2$ years (range: 0-16 years), from the Department of Neurology in the First People's Hospital of Guiyang, China, from
January 2014 to December 2015. This study was approved by the Ethics Committee of the First People's Hospital of Guiyang. Written informed consents were obtained from all participants.

Inclusion criteria were as follows: older than 40 years; magnetic resonance imaging (MRI) of brains indicating WML (varying degrees); no history of cerebrovascular diseases (including hemorrhagic and ischemic cerebrovascular diseases); and no specific diseases causing central nervous injury and related medical history, such as tumor, infection, carbon monoxide poisoning, demyelinating disease of the central nervous system, and degenerative diseases.

Exclusion criteria were the following: previously confirmed cognitive impairment; factors that affect the results of measuring cognitive function (patients with related neuropsychiatric history or depression, taking antidepressant drugs, with hypothyroidism, visual and hearing disorder, and with hemiplegia, hemidysesthesia, aphasia, and other physical signs of focal central nervous system disorders and/or cerebral hemorrhage or cerebral infarction verified with related imaging evidence); other diseases or medical history causing central nervous injuries; excessive drinking; heart, liver and kidney failure.

\section{Grouping}

All WML patients included were grouped according to different risk factors and divided into 5 groups: a no risk factor group, a hypertension group, a diabetes mellitus group, a dyslipidemia group, and a mixed factor group (including 2 or more types of the above risk factors). Hypertension referred to the patients who currently took orally antihypertensive drugs or whose multiple blood pressure values were higher than $140 / 90 \mathrm{~mm} \mathrm{Hg}$, but they were not taking medication; diabetes mellitus referred to the patients who had been diagnosed previously and/or who were currently taking insulin or oral hypoglycemic drugs for treatment; dyslipidemia referred to the patients whose total cholesterol was higher than $5.7 \mathrm{mmol} / \mathrm{L}$, low-density lipoprotein (LDL) was higher than $3.12 \mathrm{mmol} / \mathrm{L}$, high-density lipoprotein was lower than $1.20 \mathrm{mmol} / \mathrm{L}$, and triglyceride was higher than $1.88 \mathrm{mmol} / \mathrm{L}$.

Table 1. Clinical qualitative characteristics of different risk factor groups

\begin{tabular}{|l|c|c|c|c|}
\hline \multicolumn{1}{|c|}{ Variables } & No risk factor group & Hypertension group & Diabetes group & Dyslipidemia group \\
\hline Cases, $n$ & 26 & 35 & 27 & 30 \\
\hline Male/female & $10 / 16$ & $13 / 22$ & $10 / 17$ & $10 / 20$ \\
\hline Age [years] & $69.73(12.58)$ & $72.03(6.95)$ & $63.55(6.15)$ & $14 / 25$ \\
\hline Education [years] & $9.31(4.35)$ & $10.86(3.66)$ & $11.82(3.25)$ & $70.33(8.88)$ \\
\hline ADL [points] & $21.12(3.39)$ & $21.83(3.27)$ & $20.63(1.03)$ & $9.53(4.23)$ \\
\hline HAMD [points] & $4.09(1.64)$ & $4.94(1.19)$ & $5.09(1.38)$ & $20.70(1.32)$ \\
\hline
\end{tabular}

$\mathrm{ADL}$ - activities of daily living scale; HAMD - Hamilton Depression scale. 


\section{Imaging evaluation}

The degree of WML could be quantified and evaluated using MRI adopting the Fazekas scale (the lowest total score is 0 , the highest -6$).{ }^{8}$ The changes of periventricular and deep cerebral white matter were evaluated and the total scores was obtained through generalizing the scores of the 2 parts. The respective scores meant for periventricular white matter hyperintense signals: 0 - absence; 1 - cap shape or pencil-thin lining; 2 - smooth halo; 3 - irregular periventricular hyperintense signals spreading into deep white matter. For deep white matter hyperintense signals the scores meant: 0 - absence; 1 - point-like foci; 2 - starting confluence of point-like foci; 3 - large confluent areas. Cranial MRI was jointly judged by clinical neurologists and radiologists.

\section{Neuropsychological assessment}

The Montreal Cognitive Assessment (MoCA) scale and auditory verbal memory test (including immediate word recall, delayed word recall and word recognition) were used to evaluate the cognitive function. Blind operation and judgment of results were carried out by well-trained neurologists. The ADL scale was used to evaluate the general conditions of patients, while the HAMD scale was used for exclusion of patients with depression.

\section{Blood biochemical test}

Fasting venous blood was collected from the patients (fast for $8 \mathrm{~h}$ ) for analysis of liver function, renal function, blood lipids, blood glucose, uric acid, and thyroid function to monitor the general conditions of the patients.

\section{Statistical analysis}

Data analysis was performed using SPSS v. 18.0 software (SPSS Inc., Chicago, USA). The data was presented as mean \pm standard deviation (SD). Enumeration data was compared with a $X^{2}$ test. The comparison for quantitative data among multiple groups was assessed with one-way analysis of variance (ANOVA) followed by the post-hoc Bonferroni test. A p-value of less than 0.05 was considered as statistical significance.

\section{Results}

\section{Comparison of WML and cognition in different risk factor groups}

White matter lesions severity (Fazekas score) in the hypertension and mixed factors groups was higher than that in the no risk factor group ( $\mathrm{p}=0.022$; Fig. $1 \mathrm{~A}$ ); there was no statistical difference among other groups. The MoCA scores of the hypertension group and mixed factors group were lower than in the no risk factor group ( $p=0.018$; Fig. $1 B$ ) without statistical difference among the other groups. In addition, there were no statistical differences regarding the comparisons of immediate word recall, delayed word recall and word recognition among all groups (Fig. 1C-E).

\section{The effect of WML degree on cognition}

According to Fazekas scoring, WML severity can be divided into a Fazekas score $<3$ group and the studied population. Comparing the association of different WML severities with cognitive impairment in all groups, we found that the MoCA score, immediate word recall score and delayed word recall score of patients with a Fazekas score $\geq 3$ in the hypertension group and mixed factor group were lower than of those with a Fazekas score $<3$ in the same groups $(p=0.031$; Table 2$)$. In addition, we found that the MoCA score and immediate word recall score of patients with a Fazekas score $\geq 3$ in the hypertension group and mixed factor group were lower than of those with a Fazekas score $\geq 3$ in the no risk factor group ( $p=0.022$; Table 2). There were no statistical significances for the comparison of the MoCA score, immediate word
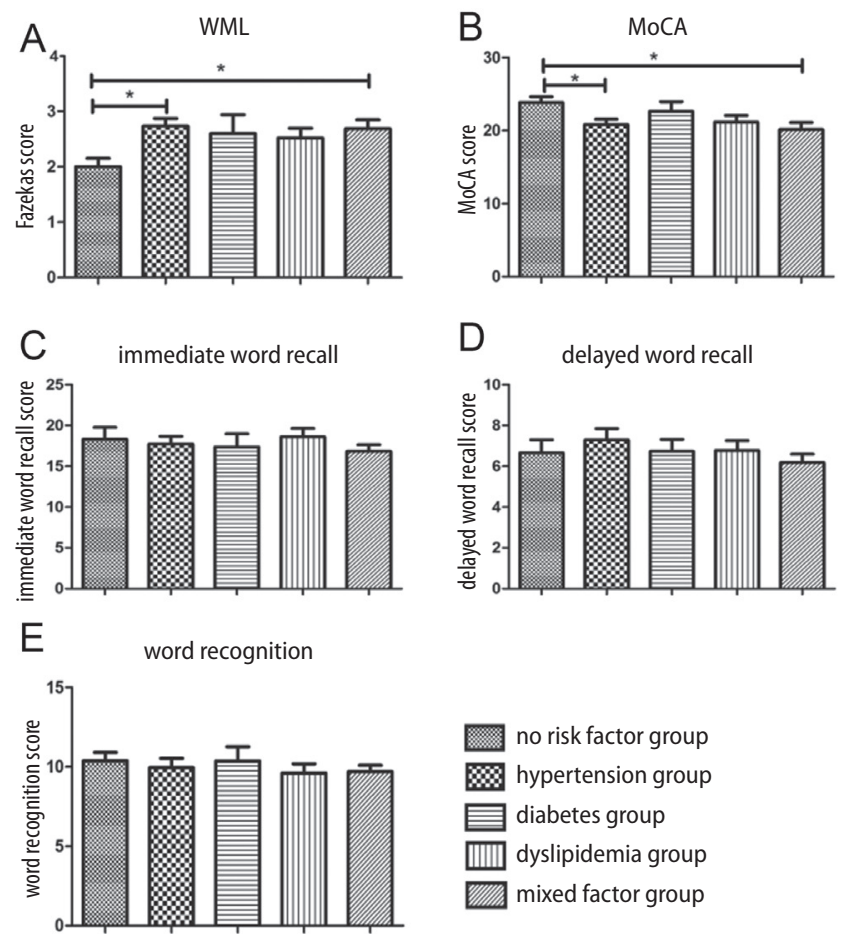

Fig. 1. Comparison of WML and cognitive scores among different risk factor groups. A. Fazekas score indicated that the WML severity of the hypertension group and mixed factors group was higher than that of the no risk factor group, and there was no statistical difference among other groups. B. MoCA scores of the hypertension group and mixed factor group were lower than that of the no risk factor group, without statistical difference among other groups. C-E. There were no statistical differences in terms of the comparisons of immediate word recall, delayed word recall and word recognition among these groups

${ }^{*} p<0.05$ - statistical significance. 
Table 2. Quantitative comparisons of different WML severities on cognition in each risk factor group

\begin{tabular}{|l|c|c|c|c|c|c|}
\multicolumn{1}{|c|}{ Group } & $\begin{array}{c}\text { Fazekas } \\
\text { score }\end{array}$ & Cases (\%) & MoCA & $\begin{array}{c}\text { Immediate word } \\
\text { recall }\end{array}$ & $\begin{array}{c}\text { Delayed word } \\
\text { recall }\end{array}$ & Word recognition \\
\hline No risk factor group & $<3$ & $20(76.9)$ & $24.05(4.26)$ & $19.64(7.09)$ & $10.88(6.96)$ & $10.77(2.37)$ \\
& $\geq 3$ & $6(23.1)$ & $22.30(7.53)$ & $18.10(4.00)$ & $5.50(1.73)$ & $8.25(3.59)$ \\
\hline \multirow{2}{*}{ Hypertension group } & $<3$ & $15(42.9)$ & $23.26(4.38)$ & $19.48(6.62)$ & $12.94(8.06)$ & $10.74(3.32)$ \\
\hline Diabetes group & $\geq 3$ & $20(57.1)$ & $18.50(3.48)^{* \#}$ & $14.67(5.10)^{* \#}$ & $5.17(2.69)^{*}$ & $8.42(3.32)$ \\
\hline \multirow{2}{*}{ Dyslipidemia group } & $\geq 3$ & $13(48.1)$ & $22.63(4.75)$ & $18.50(5.32)$ & $11.56(6.42)$ & $10.25(2.31)$ \\
\hline Mixed factor group & $\geq 3$ & $14(51.9)$ & $22.67(4.04)$ & $18.67(5.67)$ & $7.00(2.00)$ & $10.67(5.13)$ \\
\hline & $\geq 3$ & $18(60.0)$ & $22.05(3.64)$ & $19.42(6.25)$ & $11.67(7.68)$ & $9.42(3.39)$ \\
\hline
\end{tabular}

* MoCA score, immediate word recall score and delayed word recall score in the hypertension group and mixed factor group with Fazekas $\geq 3$ are lower than those in the same groups with Fazekas score $<3, \mathrm{p}<0.05$.

\# MoCA score and immediate word recall score of the hypertension group and mixed factor group with Fazekas score $\geq 3$ are lower than those of the no risk factor group with Fazekas score $\geq 3, p<0.05$. There was no statistical significance on the comparisons of MoCA, immediate word recall, delayed word recall, and word recognition among all risk factor groups with Fazekas score $<3$

recall score, delayed word recall score, and word recognition score among all risk factor groups when patients with Fazekas score $<3$ were concerned (Table 2).

\section{Discussion}

With the continuous development of neuroimaging technology, the detection rate of WML is significantly improved. The imaging description of WML is manifested with symmetricly speckled or patchy changes of periventricular and centrum ovale white matter. Magnetic resonance T2-weighted image shows high signal intensity, whereas T1-weighted image reveals equal or low signal intensity. In clinical practice, WML is a prevalent disease associated with multiple neurologic disorders, ${ }^{1}$ especially with cognitive impairment. ${ }^{9,10}$ Some risk factors of cerebrovascular diseases, such as hypertension and diabetes mellitus, can lead to the development of WML. ${ }^{5-7}$ However, the relationship between the different risk factors and cognitive impairment in consequence of WML remains unclear. This study aimed to investigate the cognitive impairment features of WML accompanied with different risk factors of cerebrovascular diseases.

\section{The influence of severity of WML on cognition}

Scott et $\mathrm{al}^{3}$ found that in aged people with normal cognition, WML is widely thought to be the sign of cerebral small vessel disease, which is associated with vascular injury caused by vascular risk factors, including hypertension, high cholesterol and diabetes mellitus. Medical history of hypertension is independently associated with WML capacity. Our study has also found that the WML (measured with Fazekas scoring) in the hypertension and mixed factor groups is more severe than in the no risk factor group. Some scholars have found that MoCA is more sensitive than Mini-Mental State Examination (MMSE) in detecting the cognitive impairment in WML patients. ${ }^{11}$ Therefore, we evaluated the patients' congnition by using MoCA scale and auditory verbal memory, and found that MoCA scores in the hypertension and mixed factor groups were lower than in the no risk factor group, and there was no statistical significance in immediate word recall, delayed word recall and word recognition among all groups. Sierra ${ }^{12}$ found that hypertensive patients are more prone to WML than normotensive people, and that arteriosclerosis of the cerebral perforator vessel is the primary cause of ischemic WML. In patients with declining cognition and dementia, chronic ischemia of white matter is associated with arteriosclerosis and/or the lipohyalinosis of small perforating artery hypertension, and antihypertensive treatment can reduce the risk of dementia. ${ }^{13}$ In the elderly populations, excessive variation of self-measured systolic blood pressure aggravates the progress of cognitive functional impairment and WML. ${ }^{14}$ The study of Peng et al. ${ }^{15}$ found that systolic blood pressure controlled within 140-160 $\mathrm{mm} \mathrm{Hg}$ and systolic blood pressure reduced by $15-35 \mathrm{~mm} \mathrm{Hg}$ are beneficial in delaying the progression of cognitive impairment and WML. Our study found that the severity of WML and MoCA score in the hypertension and mixed factor groups were statistically different from those in the no risk factor group, and there were no statistical differences between the diabetes mellitus group/dyslipidemia group and no risk factor group. However, some scholars have found that diabetes mellitus and dyslipidemia were associated with WML, ${ }^{4,16}$ leading to declined cognition ${ }^{17}$ and increased risk of vascular dementia. ${ }^{6}$ Considering the effect of diabetes mellitus and dyslipidemia in the mixed factor group, the sample size should be increased to further investigate the independent effect of diabetes mellitus and dyslipidemia on WML and cognition in subsequent research. 


\section{Fazekas score and severe WML}

The MoCA score, immediate word recall score and delayed word recall score of patients from the hypertension group and mixed factor group with Fazekas score $\geq 3$ were lower than of those from the same groups with Fazekas score $<3$. The MoCA score and immediate word recall score of patients from the hypertension group and mixed factor group with Fazekas score $\geq 3$ were lower than of those from the no risk factor group with Fazekas score $\geq 3$, but there were no statistical differences in the comparisons of those from all risk factor groups with Fazekas score $<3$. Therefore, we speculate that the severity of WML in the hypertension group and mixed factors group is positively correlated with cognitive functional impairment, and that the cognitive impairment in WML with risk factors is more severe than that in WML with no risk factors. Defrancesco et al. ${ }^{18}$ have found that patients with mild cognitive impairment (MCI) converting to Alzheimer's disease (AD) obtain higher periventricular Fazekas scores and present more severe WML. Periventricular WML is associated with low cognitive function of MCI patients, which is consistent with the findings of our study. The severity of WML accelerates the progression of MCI. ${ }^{19}$ Maillard et al. ${ }^{20}$ found that increased WML is obviously related to the decline of episodic memory and executive function, and the progression of WML is related to cognition. Some studies have shown that the more severe the damage to periventricular white matter is, the higher the risk of dementia, ${ }^{21}$ and cognitive impairment of WML is associated with the severity of WML. ${ }^{22}$ The white matter is mainly supplied by the vertical short branch of the terminal artery with less anastomotic branches and poor collateral circulation. ${ }^{16}$ Therefore, the blood flow volume in the white matter is lower than in the grey matter. In addition, the risk factors of cerebrovascular diseases cause damage in small cerebral blood vessels, so less cerebral blood flow and insufficient cerebral perfusion will lead to ischemic injury in the white matter. Therefore, the cognitive impairment of WML accompanied with the risk factors of cerebrovascular diseases may be more severe than in WML with no risk factors.

\section{Effect of risk factors on severity of WML}

The MoCA scale includes the detection of multiple aspects of cognitive impairment such as memory, execution, language, attention, and orientation. Auditory verbal memory is an extension of the memory test. Our study found that MoCA, immediate word recall and delayed word recall were influenced by the WML severity in the hypertension group and mixed factor group, and MoCA and immediate word recall in the hypertension group and mixed factor group were statistically different than in no risk factor group, suggesting that WML had comprehensive effects on cognition. Te et al. ${ }^{23}$ believed that WML patients with
MCI obviously presented the declining of memory and attention, damage of executive function and close connection with dementia in the early days. $\mathrm{Zi}$ et al. ${ }^{24}$ found out that a cognitive test for patients with periventricular high signal lesion shows obvious declining of word fluency and executive function. In addition, Vasquez et al. ${ }^{25}$ found that the processing speed and executive function in VCI are poorer. White matter lesions are an early predictive index of dementia risk, but this association is dependent on cognitive reserve, age and spatial distribution of the lesions. ${ }^{26}$ Some studies have shown that the brain white matter of the elderly was obviously less smaller in volume than that of a middle-aged group, and the brain white matter of the middle-aged was obviously less smaller in volume than that of a youth group. ${ }^{7}$ A low education level group ( $\leq 8$ years of education) presented increased risk of severe WML developing into MCI and dementia, but there were no such risks in a high education level group ( $>8$ years of education). ${ }^{27}$ In our study, there were no significant differences in age and years of education among all the groups, and the effect of these factors upon the study results was not considered.

\section{Effect of WML on automatic activity}

Severe WML results in a more than twofold increase of the risk of transition from automatic activities to activity dependence. White matter lesions are associated with a decline of cognitive and athletic ability, depressive symptoms related to aging and vascular diseases, dysfunction of the urinary system, and various abnormities of the nervous system. ${ }^{28}$ Such lesions are the primary cause of falls. ${ }^{29,30}$ Al-Mashhadi et al. ${ }^{31}$ found that the dysfunction of WML is not confined to lesions, and that the normal white matter is also damaged. In view of the perniciousness of WML, we need to identify and impede the risk factors, aiming to prevent the progression and improve cognitive prognosis. A study found that L-carnitine can improve WML and prevent cognitive impairment of a chronic hypo-perfusion model, ${ }^{32}$ and another study found that supplementation of 6-g L-arginine in diet is beneficial to improving the cognition and preventing gait disorders of WML patients. ${ }^{33}$

\section{Study limitation}

In this study, the interaction between variables was not considered and analyzed, which might affect the statistical results. We will consider and analyze this point in further investigation.

\section{Conclusions}

Hypertension and multiple risk factors of cerebrovascular diseases will aggravate the severity of WML and cognitive impairment, and the severity of WML accompanied 
with these risk factors is positively correlated with the degree of cognitive impairment. However, the damage of cognitive domains of WML affected with WML accompanied by different risk factors of cerebrovascular diseases may be different. Therefore, we plan to expand the sample size to compare execution, attention, language, orientation, and other cognitive domains in the future. We need to further investigate the cognitive features of WML accompanied by diabetes mellitus and dyslipidemia, aiming to comprehensively understand the features of cognitive impairment in WML accompanied by different risk factors of cerebrovascular diseases.

\section{ORCID iDs}

Yafei Shangguan (1) https://orcid.org/0000-0002-5272-1972 Tao Xiong (10 https://orcid.org/0000-0001-8247-8582 Changwei Jiang (1) https://orcid.org/0000-0001-9728-6209 Wei Chen (10 https://orcid.org/0000-0003-0171-194X Yan Zhang (1) https://orcid.org/0000-0002-3566-2907 Yongpin Zhao (1) https://orcid.org/0000-0003-2979-1478 Guiyin Zhou (1) https://orcid.org/0000-0003-2870-1178 Yulan Fan (10) https://orcid.org/0000-0002-7021-0770 Weimin Liu (1] https://orcid.org/0000-0001-6966-0101

\section{References}

1. O'Brien JT. Clinical significance of white matter changes. Am J Geriatr Psychiatry. 2014;22(2):133-137.

2. Miki Y, Sakamoto $S$. Age-related white matter lesions (leukoaraiosis): An update [in Japanese]. Brain Nerve. 2013;65(7):789-799.

3. Scott JA, Braskie MN, Tosun D, et al; Alzheimer's Disease Neuroimaging Initiative. Cerebral amyloid and hypertension are independently associated with white matter lesions in elderly. Front Aging Neurosci. 2015;7:221.

4. Bowler JV. The concept of vascular cognitive impairment. J Neurol Sci. 2002;203-204:11-15.

5. Akiguchi I, Yamamoto Y. Vascular mechanisms of cognitive impairment: Roles of hypertension and subsequent small vessel disease under sympathetic influences. Hypertens Res. 2010;33(1):29-31.

6. Xu W, Qiu C, Gatz M, Pedersen NL, Johansson B, Fratiglioni L. Midand late-life diabetes in relation to the risk of dementia: A population-based twin study. Diabetes. 2009;58(1):71-77.

7. Brickman AM, Zimmerman ME, Paul RH, et al. Regional white matter and neuropsychological functioning across the adult lifespan. Biol Psychiatry. 2006;60(5):444-453.

8. Fazekas F, Chawluk JB, Alavi A, Hurtig HI, Zimmerman RA. MR signal abnormalities at $1.5 \mathrm{~T}$ in Alzheimer's dementia and normal aging. AJR Am J Roentgenol. 1987;149(2):351-356.

9. Prins ND, van Dijk EJ, den Heijer T, et al. Cerebral small-vessel disease and decline in information processing speed, executive function and memory. Brain. 2005;128(Pt 9):2034-2041.

10. Benedictus MR, van Harten AC, Leeuwis AE, et al. White matter hyperintensities relate to clinical progression in subjective cognitive decline. Stroke. 2015;46(9):2661-2664.

11. Li J, Hu W. Glucose metabolism measured by positron emission tomography is reduced in patients with white matter presumably ischemic lesions. Med Sci Monit. 2014;20:1525-1530.

12. Sierra C. Essential hypertension, cerebral white matter pathology and ischemic stroke. Curr Med Chem. 2014;21(19):2156-2164.

13. Hanon O. Hypertension and dementia [in French]. Ann Cardiol Angeiol (Paris). 2014;63(3):204-208.
14. Liu Z, Zhao Y, Zhang H, et al. Excessive variability in systolic blood pressure that is self-measured at home exacerbates the progression of brain white matter lesions and cognitive impairment in the oldest old. Hypertens Res. 2016;39(4):245-253.

15. Peng J, Lu F, Wang Z, et al. Excessive lowering of blood pressure is not beneficial for progression of brain white matter hyperintensive and cognitive impairment in elderly hypertensive patients: 4-year followup study. J Am Med Dir Assoc. 2014;15(12):904-910.

16. Tullberg $M$, Fletcher $E$, DeCarli $C$, et al. White matter lesions impair frontal lobe function regardless of their location. Neurology. 2004; 63(2):246-253.

17. Williamson JD, Launer LJ, Bryan RN, et al; Action to Control Cardiovascular Risk in Diabetes Memory in Diabetes Investigators. Cognitive function and brain structure in persons with type 2 diabetes mellitus after intensive lowering of blood pressure and lipid levels: A randomized clinical trial. JAMA Intern Med. 2014;174(3):324-333.

18. Defrancesco M, Marksteiner J, Deisenhammer E, Kemmler G, Djurdjevic T, Schocke M. Impact of white matter lesions and cognitive deficits on conversion from mild cognitive impairment to Alzheimer's disease. J Alzheimers Dis. 2013;34(3):665-672.

19. Devine ME, Fonseca JA, Walker Z. Do cerebral white matter lesions influence the rate of progression from mild cognitive impairment to dementia? Int Psychogeriatr. 2013;25(1):120-127.

20. Maillard P, Carmichael O, Fletcher E, Reed B, Mungas D, DeCarli C. Coevolution of white matter hyperintensities and cognition in the elderly. Neurology. 2012;79(5):442-448.

21. Prins ND, van Dijk EJ, den Heijer T, et al. Cerebral white matter lesions and the risk of dementia. Arch Neurol. 2004;61(10):1531-1534.

22. Loeb C, Gandolfo C, Croce R, Conti M. Dementia associated with lacunar infarction. Stroke. 1992;23(9):1225-1229.

23. Te M, Zhao E, Zheng X, Sun Q, Qu C. Leukoaraiosis with mild cognitive impairment. Neurol Res. 2015;37(5):410-414.

24. Zi W, Duan D, Zheng J. Cognitive impairments associated with periventricular white matter hyperintensities are mediated by cortical atrophy. Acta Neurol Scand. 2014;130(3):178-187.

25. Vasquez BP, Zakzanis KK. The neuropsychological profile of vascular cognitive impairment not demented: A meta-analysis. J Neuropsychol. 2015;9(1):109-136.

26. Mortamais M, Artero S, Ritchie K. Cerebral white matter hyperintensities in the prediction of cognitive decline and incident dementia. Int Rev Psychiatry. 2013;25(6):686-698.

27. Mortamais $M$, Portet $F$, Brickman AM, et al. Education modulates the impact of white matter lesions on the risk of mild cognitive impairment and dementia. Am J Geriatr Psychiatry. 2014;22(11):1336-1345.

28. Pantoni L, Fierini F, Poggesi A. Impact of cerebral white matter changes on functionality in older adults: An overview of the LADIS Study results and future directions. Geriatr Gerontol Int. 2015;15(Suppl 1): 10-16.

29. Morley JE. White matter lesions (leukoaraiosis): A major cause of falls. J Am Med Dir Assoc. 2015;16(6):441-443.

30. Ogama N, Sakurai T, Shimizu A, Toba K. Regional white matter lesions predict falls in patients with amnestic mild cognitive impairment and Alzheimer's disease. J Am Med Dir Assoc. 2014;15(1):36-41.

31. Al-Mashhadi S, Simpson JE, Heath PR, et al; Medical Research Council Cognitive Function and Ageing Study. Oxidative glial cell damage associated with white matter lesions in the aging human brain. Brain Pathol. 2015;25(5):565-574.

32. Ueno $Y$, Koike M, Shimada Y, et al. L-carnitine enhances axonal plasticity and improves white-matter lesions after chronic hypoperfusion in rat brain. J Cereb Blood Flow Metab. 2015;35(3):382-391.

33. Calabro RS, Gervasi G, Baglieri A, Furnari A, Marino S, Bramanti P. Is high oral dose L-arginine intake effective in leukoaraiosis? Preliminary data, study protocol and expert's opinion. Curr Aging Sci. 2013; 6(2):170-177. 